\title{
Non-Functioning Pituitary Gland Microadenoma
}

National Cancer Institute

\section{Source}

National Cancer Institute. Non-Functioning Pituitary Gland Microadenoma. NCI

Thesaurus. Code C121678.

A pituitary gland microadenoma not associated with a hormonal syndrome. 